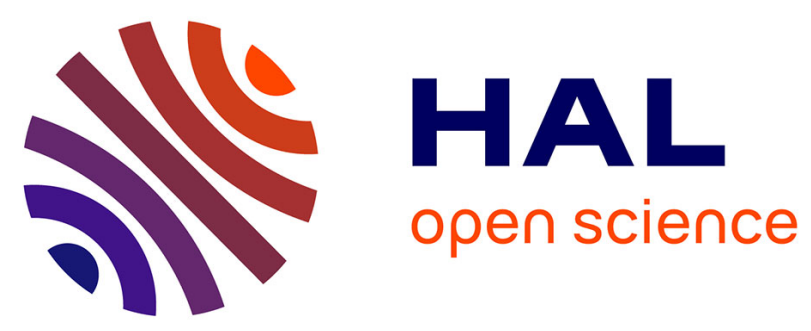

\title{
Coupling tabulated chemistry with Large Eddy Simulation of turbulent reactive flows
}

Ronan Vicquelin, Benoit Fiorina, Nasser Darabiha, Olivier Gicquel, Denis Veynante

\section{- To cite this version:}

Ronan Vicquelin, Benoit Fiorina, Nasser Darabiha, Olivier Gicquel, Denis Veynante. Coupling tabulated chemistry with Large Eddy Simulation of turbulent reactive flows. Comptes Rendus Mécanique, 2009, 337 (6-7), pp.329 - 339. 10.1016/j.crme.2009.06.011 . hal-01744304

\section{HAL Id: hal-01744304 https://hal.science/hal-01744304}

Submitted on 27 Mar 2018

HAL is a multi-disciplinary open access archive for the deposit and dissemination of scientific research documents, whether they are published or not. The documents may come from teaching and research institutions in France or abroad, or from public or private research centers.
L'archive ouverte pluridisciplinaire HAL, est destinée au dépôt et à la diffusion de documents scientifiques de niveau recherche, publiés ou non, émanant des établissements d'enseignement et de recherche français ou étrangers, des laboratoires publics ou privés. 


\title{
Coupling Tabulated Chemistry with Large Eddy Simulation of Turbulent Reactive Flows
}

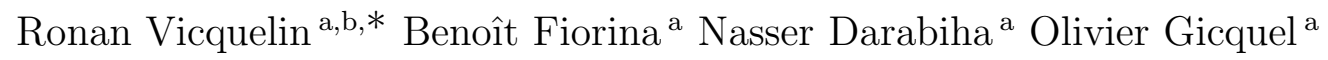 \\ Denis Veynante ${ }^{\mathrm{a}}$ \\ ${ }^{a}$ Laboratoire EM2C-CNRS, Ecole Centrale Paris 92290 Châtenay Malabry, France \\ ${ }^{\mathrm{b}}$ GDF SUEZ, Pôle CHENE, Centre de Recherche et d'Innovation Gaz et Energies Nouvelles, 93211 Saint-Denis la \\ Plaine, France
}

\begin{abstract}
A new modeling strategy is developed to introduce tabulated chemistry methods in LES of turbulent premixed combustion. The objective is to recover the correct laminar flame propagation speed of the filtered flame front when subgrid scale turbulence vanishes. The filtered flame structure is mapped by $1 \mathrm{D}$ filtered laminar premixed flames. Closure of the filtered progress variable and the energy balance equations are carefully addressed. The methodology is applied to $1 \mathrm{D}$ and 2D filtered laminar flames. These computations show the capability of the model to recover the laminar flame speed and the correct chemical structure when the flame wrinkling is completely resolved. The model is then extended to turbulent combustion regimes by introducing subgrid scale wrinkling effects on the flame front propagation. Finally LES of a 3D turbulent premixed flame is performed.
\end{abstract}

\section{Résumé}

Couplage entre chimie tabulée et simulation aux grandes échelles. Un nouveau modèle est développé pour utiliser des méthodes de chimie tabulée dans la simulation aux grandes échelles de la combustion turbulente prémélangée. Le but est de retrouver la vitesse de propagation de flamme laminaire pour une flamme filtrée lorsque la turbulence en sous maille est négligeable. La structure de la flamme filtrée est reproduite par une flamme laminaire unidimensionnelle filtrée. Un soin particulier est porté à la fermeture des équations de transport de la variable d'avancement de réaction filtrée et d'énergie filtrée. Le modèle est appliqué à des cas unidimensionnelle et bidimensionnelle de flamme laminaire. Ces calculs montrent que la vitesse de flamme et sa structure chimique sont correctement reproduites lorsque le plissement de la flamme est résolu. Le modèle est ensuite étendu à la combustion turbulente en prenant en compte l'effet du plissement de la flamme en sous-maille sur sa propagation. Une simulation aux grandes échelles d'une flamme turbulente tridimensionnelle est finalement réalisée.

Key words: Large eddy simulation; turbulent premixed combustion ; tabulated chemistry ; filtered flame

Mots-clés : Simulation aux grandes échelles; combustion turbulente prémélangée; flamme filtrée 


\section{Introduction}

Flame ignition and extinction or pollutant prediction are crucial issues in LES of premixed combustion and are strongly influenced by chemical effects. Unfortunately, despite the rapid increase in computational power, to perform turbulent simulations of industrial configurations including detailed mechanisms remains out of reach. A commonly used approach to tackle fluid/chemistry interactions at a reduced computational cost consists of tabulating chemistry effectively assessed in a realistic turbulent flow. Some techniques, such as ILDM developed by Mass \& Pope [1], are based on a direct mathematical analysis of the dynamical behavior of the chemical system response. Alternatives that requires less dimensions are FPI [2,3] or FGM [4]. Both techniques assume that the chemical flame structure can be described in a reduced phase subspace from elementary combustion configurations. For instance, the chemical subspace of turbulent premixed configuration can be approximated from a 1D laminar flame. In such simple situations, all thermo-chemical quantities are related to a single progress variable. Turbulent combustion models have been provided in the past for RANS $[5,6]$ but extension of tabulated chemistry to LES presents additional challenges.

The primary recurrent problem is that the flame thickness is typically thinner than the LES grid size. As the progress variable source term is very stiff, the flame front field cannot be directly resolved in LES, leading to numerical issues. To overcome this difficulty, dedicated models have been developed in the past under simplified chemistry assumptions. A solution to propagate a flame on a coarse grid is to artificially thicken the flame front by modifying diffusion coefficient and preexponential constant $[7,8]$. Following a different strategy, Boger et al. [9] and more recently Duwig et al. [10] have introduced a filter larger than the mesh size to resolve the filtered flame structure. An opposite alternative to enlarge the flame front is the G-equation model where the inner layer is tracked using a level-set technique. Initially developed in a RANS context, the G-equation has been reformulated by Pitsch [11] for LES. Recently Moureau et al. [12] have introduced a progress variable to ensure a consistent coupling with a LES flow solver but under a simplified chemistry assumption.

The FPI-PCM model has been developed to introduce tabulated chemistry effects in LES [13]. It combines presumed PDF and FPI tables to close the filtered progress variable chemical reaction rate and to model interactions between turbulence and chemistry at the subgrid scale level. However, as shown further, this formulation does not guarantee a proper degeneration to the laminar flame regime when subgrid scale turbulence vanishes. This regime, observed when the subgrid fluctuations are lower than the laminar flame speed [14], is encountered in practical LES of premixed combustion [12].

The capability of presumed $\beta$-PDF to reproduce such regimes where wrinkling entirely occurs at the resolved scale, is accessed conducting a priori tests on a 1D stoichiometric freely propagating propane/air laminar flame computed with GRI 3.0 mechanism (http://www.berkeley.edu/gri_mech/). The progress variable, $c$, is plotted as a function of the spatial coordinate in Fig. 1(a). A reference filtered flame solution is obtained by applying a Gaussian filter $F$ of size $\Delta=100 \delta_{l}$, where $\delta_{l}$ is the laminar flame thickness. Favre-filtered progress variable $\widetilde{c}$ and the segregation factor $S_{c}=\widetilde{c^{\prime \prime 2}} /(\widetilde{c}(1-\widetilde{c}))$ are shown on Fig. 1(a). The presumed $\beta$-PDF $\widetilde{P_{\beta}}\left(c=c^{*}\right)$ is then computed from these two quantities and compared to the exact density weighted probability density function $\widetilde{P}\left(c=c^{*}\right)$ given by its definition [15] in the case of a filtered 1D laminar flame by the following expression:

\footnotetext{
*. Corresponding author.

Email addresses: ronan.vicquelin@em2c.ecp.fr (Ronan Vicquelin), benoit.fiorina@em2c.ecp.fr (Benoît Fiorina), nasser.darabiha@em2c.ecp.fr (Nasser Darabiha), olivier.gicquel@em2c.ecp.fr (Olivier Gicquel), denis.veynante@em2c.ecp.fr (Denis Veynante).
} 


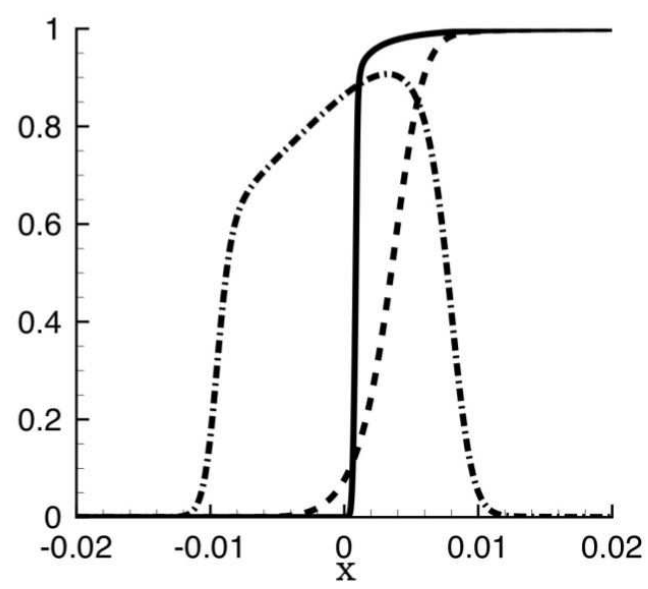

(a)

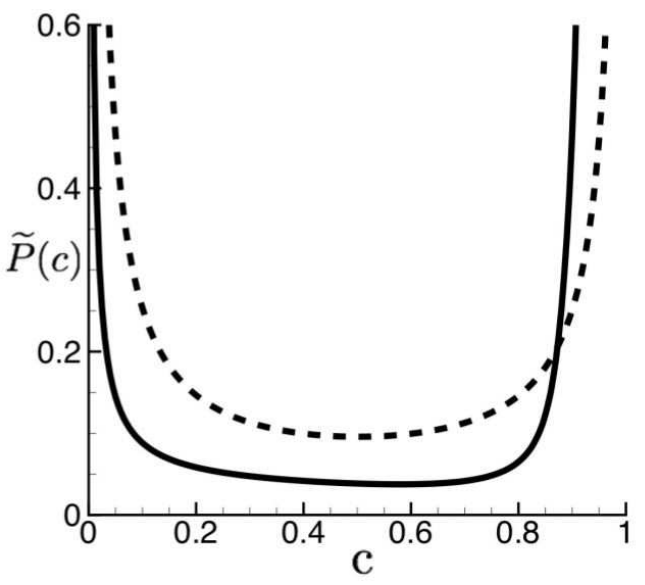

(b)

Figure 1. A priori test of the $\beta$-PDF in laminar regime. Left (a): progress variable $c$ (solid line) and filtered progress variable $\widetilde{c}$ (dashed line) profiles as a function of the spatial coordinate $x$. Dashed dotted line is the subfilter progress variable segregation factor $S_{c}=\widetilde{c^{\prime \prime 2}} /(\widetilde{c}(1-\widetilde{c}))$. Right (b): exact probability density function (PDF) of the progress variable (solid line) compared with the presumed $\beta$-PDF (dashed line) at $\widetilde{c}=0.5$

$$
\widetilde{P}\left(c^{*}, x\right)=\frac{1}{\bar{\rho}} \int_{-\infty}^{+\infty} \rho\left(x^{\prime}\right) \delta\left(c\left(x^{\prime}\right)-c^{*}\right) F\left(x-x^{\prime}\right) d x^{\prime}=\alpha \delta\left(c^{*}\right)+\frac{\rho\left(c^{*}\right) F\left(x-x\left(c^{*}\right)\right)}{\left.\bar{\rho} \frac{\partial c}{\partial x}\right|_{x=x\left(c^{*}\right)}}+\beta \delta\left(1-c^{*}\right)
$$

where $\delta$ is the Dirac function, $\rho$ the mass density, $\mathrm{F}$ the filter function, $\alpha$ and $\beta$ weight coefficients. Figure 1 (b) shows that the progress variable probability density function differs from the $\beta$ function. Integration of the filtered reaction rate over the flame brush shows that the propagation speed of the filtered progress variable is over-estimated with the presumed $\beta$ function by a factor of about 2.5 .

In the present work, an alternative to the $\beta$-PDF formalism is proposed to include tabulated chemistry in LES and to ensure the correct propagation speed of the filtered flame front. It is assumed that in a an LES context, the flame structure can be mapped from a one-dimensional filtered premixed flame. Closure of filtered flow and progress variable equations are first carefully addressed in the laminar regime. Onedimensional and two-dimensional computations are then performed to investigate the capability of the proposed model to reproduce the correct propagation speed and the filtered flame structure. The model is then extended to turbulent combustion regimes with the introduction of a flame wrinkling factor. Finally simulations of a turbulent premixed flame stabilized by a triangular flame holder are performed.

\section{Governing equations}

The chemical evolution of the reactive flow is described by using a progress variable $c$ related to temperature or fuel mass fraction or a combination of other species, where $c=0$ corresponds to fresh gases and $c=1$ to fully burnt gases. A transport equation is then written for the filtered progress variable coupled with the Navier-Stokes equations. The system of filtered equations reads:

$$
\frac{\partial \bar{\rho}}{\partial t}+\nabla \cdot(\bar{\rho} \widetilde{\mathbf{u}})=0
$$




$$
\begin{aligned}
\frac{\partial \bar{\rho} \widetilde{\mathbf{u}}}{\partial t}+\nabla \cdot(\bar{\rho} \widetilde{\mathbf{u}})= & -\nabla \cdot(\bar{P} \underline{\delta})+\nabla \cdot \bar{\tau}-\nabla \cdot(\bar{\rho} \widetilde{\mathbf{u} u}-\bar{\rho} \widetilde{\mathbf{u}} \widetilde{\mathbf{u}}) \\
\frac{\partial \bar{\rho} \widetilde{c}}{\partial t}+\nabla \cdot(\bar{\rho} \widetilde{\mathbf{u}} \widetilde{c})= & \nabla \cdot(\overline{\rho D \nabla c})-\nabla \cdot(\bar{\rho} \widetilde{\mathbf{u}}-\bar{\rho} \widetilde{\mathbf{u}} \widetilde{c})+\overline{\dot{\omega}}_{c} \\
\frac{\partial \bar{\rho} \widetilde{E}}{\partial t}+\nabla \cdot(\bar{\rho} \widetilde{\mathbf{u}} \widetilde{E})= & -\nabla \cdot(\overline{P \mathbf{u}} \underline{)})+\nabla \cdot(\bar{\tau} \overline{\mathbf{u}})-\nabla \cdot(\bar{\rho} \widetilde{\mathbf{u} E}-\bar{\rho} \widetilde{\mathbf{u}} \widetilde{E}) \\
& +\nabla \cdot(\overline{\rho D \nabla h})+\overline{\dot{\omega}}_{E} \\
\bar{P}= & \bar{\rho} r \widetilde{T}
\end{aligned}
$$

where $\rho$ is the density, $\mathbf{u}$ the velocity vector, $P$ the pressure, $\underline{\delta}$ the unit tensor, $\tau$ the laminar viscous tensor, E the total non-chemical energy, $h$ the sensible enthalpy, $D$ is the diffusivity, $\dot{\omega}_{c}$ and $\dot{\omega}_{T}$ respectively the chemical and energy source terms and $r$ the ideal gas constant. Note that unity Lewis number is assumed. The overbar denotes the spatial filtering operation,

$$
\bar{\phi}(\mathbf{x})=\int F\left(\mathbf{x}-\mathbf{x}^{\prime}\right) \phi\left(\mathbf{x}^{\prime}\right) d \mathbf{x}^{\prime},
$$

where $\phi$ represents reactive flow variables and velocity components. The tilde operator denotes the densityweighted filtering defined by $\bar{\rho} \widetilde{\phi}=\overline{\rho \phi}$. For one-dimensional case, a Gaussian filter with a filter size $\Delta$ is retained:

$$
F(x)=\left(\frac{6}{\pi \Delta^{2}}\right)^{1 / 2} e^{-\frac{6 x^{2}}{\Delta^{2}}} .
$$

The subgrid scale terms, $\nabla \cdot(\bar{\rho} \widetilde{\mathbf{u u}}-\bar{\rho} \widetilde{\mathbf{u}} \widetilde{\mathbf{u}})$ and $\nabla \cdot(\bar{\rho} \widetilde{\mathbf{u}} \widetilde{\varphi}-\bar{\rho} \widetilde{\mathbf{u} \varphi})$, where $\varphi$ denotes $c$ or $E$ quantities, as well as the filtered laminar diffusion terms $\overline{\rho D \nabla \varphi}$ and the filtered source terms $\overline{\dot{\omega}}_{\varphi}$, require closure models. In the present analysis, the local chemical flame structure is identified to the one of a one-dimensional laminar premixed flame (flamelet assumption).

The objective of the model proposed in the present work is both to ensure a correct flame propagation and to recover the chemical structure of the filtered flame. Two situations are considered: (1) the flame wrinkling is fully resolved at the LES filter size, and (2) wrinkling occurs at the subgrid scale and affects the filtered flame speed.

\section{Laminar filtered premixed flames modeling}

\subsection{Mathematical model}

The flame structure in the direction $\mathbf{n}$ normal to the flame front, is assumed identical to the structure of a one-dimensional freely-propagating laminar premixed flame. A one-dimensional laminar flame computed with detailed chemistry, including $N_{s}$ species, is used as reference. For a given filter size $\Delta$, filter operators introduced in Section 2 are applied to thermo-chemical quantities such as species mass fractions $Y_{i},(i=$ $\left.1, N_{s}\right)$, and source terms $\dot{\omega}_{i}$.

\section{Filtered chemical reaction rates}

These quantities are remapped in the $c$ coordinate in order to constitute a filtered flame database for a given filter size $\Delta$. Then, filtered source terms for $c$ and the energy equations are given by:

$$
\overline{\dot{\omega}}_{\varphi}=\overline{\dot{\omega}}_{\varphi}(\widetilde{c}),
$$


Filtered laminar diffusion terms $\nabla \cdot(\overline{\rho D \nabla c})$ and $\nabla \cdot(\overline{\rho D \nabla h})$

Many authors often neglect or approximate these terms in LES reactive flow solvers as:

$$
\nabla \cdot(\overline{\rho D \nabla \varphi}) \approx \nabla \cdot(\overline{\rho D} \nabla \widetilde{\varphi})
$$

However as shown further, this approximation is very rough and introduces large errors. In the present work, the filtered diffusion term for the $c$-equation is modeled by:

$$
\nabla \cdot(\overline{\rho D \nabla c}) \approx \nabla \cdot(\overline{\rho D|\nabla c|} \mathbf{n})=\nabla \cdot\left(\overline{\rho D\left|\frac{\partial c}{\partial n}\right|} \mathbf{n}\right)=\nabla \cdot\left(\alpha_{c}(\widetilde{c}) \overline{\rho D} \nabla \widetilde{c}\right)
$$

where the correction factor $\alpha_{c}(\widetilde{c})$, tabulated as a function of $\widetilde{c}$, is defined as:

$$
\alpha_{c}(\widetilde{c})=\frac{\overline{\rho D\left|\frac{\partial c}{\partial n}\right|}}{\overline{\rho D}\left|\frac{\partial \widetilde{c}}{\partial n}\right|}
$$

Similarly, the energy filtered laminar diffusion term is written as:

$$
\nabla \cdot(\overline{\rho D \nabla h})=\nabla \cdot\left(\alpha_{E}(\widetilde{c}) \overline{\rho D} \nabla \widetilde{h}\right) .
$$

Subgrid scale convection terms $\nabla \cdot(\bar{\rho} \widetilde{\mathbf{u}} \widetilde{\varphi}-\bar{\rho} \widetilde{\mathbf{u} \varphi})$

Assuming a steady state flamelet in the flame front coordinate system, $\mathbf{u}$ is decomposed as $\mathbf{u}=\mathbf{w}+\mathbf{U}$ where $\mathbf{U}$ is the fresh gases velocity and $\mathbf{w}$, the local velocity relative to the fresh gases velocity.

$$
\Omega_{\varphi}(\widetilde{\widetilde{c}})=-\nabla \cdot(\bar{\rho} \widetilde{\mathbf{u} \varphi}-\bar{\rho} \widetilde{\mathbf{u}} \widetilde{\varphi})=-\nabla \cdot(\bar{\rho} \widetilde{\mathbf{w} \varphi})-\nabla \cdot(\bar{\rho} \widetilde{\mathbf{U} \varphi})+\nabla \cdot(\bar{\rho} \widetilde{\mathbf{w}} \widetilde{\varphi})+\nabla \cdot(\bar{\rho} \widetilde{\mathbf{U}} \widetilde{\varphi})
$$

$\mathbf{U}$ remains constant across the flame brush $(\widetilde{\mathbf{U}}=\overline{\mathbf{U}}=\mathbf{U})$ and neglecting curvature effects $(\nabla \cdot \mathbf{n}=0)$ lead to:

$$
-\nabla \cdot(\bar{\rho} \widetilde{\mathbf{u} \varphi}-\bar{\rho} \widetilde{\mathbf{u}} \widetilde{\varphi})=-\nabla \cdot(\bar{\rho} \widetilde{\mathbf{w} \varphi})+\nabla \cdot(\bar{\rho} \widetilde{\mathbf{w}} \widetilde{\varphi})
$$

The laminar flame speed $S_{l}$ and the fresh gas mixture density $\rho_{0}$ are introduced:

$$
\nabla \cdot(\bar{\rho} \widetilde{\mathbf{w}} \widetilde{\varphi})=\rho_{0} S_{l} \nabla \cdot(\widetilde{\varphi} \mathbf{n}) \quad \text { and } \quad \nabla \cdot(\widetilde{\rho} \widetilde{\mathbf{w} \varphi})=\nabla \cdot\left(\overline{\rho_{0} S_{l} \mathbf{n} \varphi}\right)=\rho_{0} S_{l} \nabla \cdot(\bar{\varphi} \mathbf{n})
$$

Then, $\Omega_{\widetilde{c}}(\widetilde{\varphi})$ is tabulated according to:

$$
\Omega_{\varphi}(\widetilde{c})=\rho_{0} S_{l}\left(\left|\frac{\partial \widetilde{\varphi}}{\partial n}\right|-\left|\frac{\partial \bar{\varphi}}{\partial n}\right|\right)
$$

\section{Pressure term}

In a similar way, the pressure term in the energy equation is written as:

$$
-\nabla \cdot(\overline{P u} \underline{\delta})=-\nabla \cdot(\bar{P} \widetilde{u} \underline{\delta})+\Omega_{p}(\widetilde{c})
$$

with

$$
\Omega_{p}(\widetilde{c})=\rho_{0} S_{l}\left(\left|\frac{\partial(\widetilde{r T})}{\partial n}\right|-\left|\frac{\partial(\overline{r T})}{\partial n}\right|\right) .
$$

\section{Momentum equations}


Unclosed terms in the filtered momentum equations may be modeled following the same approach. The subgrid scale convection term and the strain tensor are written as:

$$
-\nabla \cdot(\bar{\rho} \widetilde{\mathbf{u u}}-\bar{\rho} \widetilde{\mathbf{u}})=\Omega_{u}(\widetilde{c}) \mathbf{n} \quad \text { with } \quad \Omega_{u}(\widetilde{c})=\rho_{0} S_{l}\left(\left|\frac{\partial \widetilde{u}_{n}}{\partial n}\right|-\left|\frac{\partial \bar{u}_{n}}{\partial n}\right|\right)
$$

and,

$$
\nabla \cdot \bar{\tau}=\nabla \cdot\left(\alpha_{u}(\widetilde{c}) \widetilde{\tau}\right) \quad \text { with } \quad \alpha_{u}(\widetilde{c})=\frac{\bar{\tau}_{n}}{\widetilde{\tau}_{n}}
$$

However, as will be shown, the influence of these terms is moderate and can be neglected

\subsection{Summary of the model equations}

The momentum, the progress variable and the energy equations are modeled as:

$$
\begin{aligned}
\frac{\partial \bar{\rho} \widetilde{\mathbf{u}}}{\partial t}+\nabla \cdot(\bar{\rho} \widetilde{\mathbf{u}}) & =-\nabla \cdot(\bar{P} \underline{\delta})+\nabla \cdot\left(\alpha_{u}(\widetilde{c}) \widetilde{\tau}\right)+\Omega_{u}(\widetilde{c}) \mathbf{n} \\
\frac{\partial \bar{\rho} \widetilde{c}}{\partial t}+\nabla \cdot(\bar{\rho} \widetilde{\mathbf{u}} \widetilde{c})= & \nabla \cdot\left(\alpha_{c}(\widetilde{c}) \overline{\rho D} \nabla \widetilde{c}\right)+\Omega_{c}(\widetilde{c})+\overline{\dot{\omega}}_{c}(\widetilde{c}) \\
\frac{\partial \bar{\rho} \widetilde{E}}{\partial t}+\nabla \cdot(\bar{\rho} \widetilde{\mathbf{u}} \widetilde{E})= & -\nabla \cdot(\bar{P} \widetilde{u} \underline{\delta})+\Omega_{p}(\widetilde{c})+\nabla \cdot\left(\alpha_{u}(\widetilde{c}) \tilde{\tau} \tilde{\mathbf{u}}\right)+\Omega_{E}(\widetilde{c}) \\
& +\nabla \cdot\left(\alpha_{E}(\widetilde{c}) \overline{\rho D} \nabla \widetilde{h}\right)+\overline{\dot{\omega}}_{E}(\widetilde{c})
\end{aligned}
$$

These equations are implemented into the compressible LES code AVBP [16]. The third-order finite element scheme TTGC [17] is used. Navier-Stokes characteristic boundary conditions [18] are prescribed at inlet and outlet boundary conditions.

A stoichiometric premixed 1D laminar propane/air flame is computed taking into account differential diffusion effects. The PREMIX [19] solver is combined with a modified version of the GRI 3.0 mechanism (http://www.berkeley.edu/gri_mech/) involving 70 species and 463 elementary reactions. The filtered operator given by Eq. 8 is then applied to the 1D laminar flame solution in order to evaluate the filtered chemical reactions rates $\overline{\dot{\omega}}_{\varphi}$, the diffusion fluxes correction factors $\alpha_{\varphi}$ and the subgrid scales fluxes $\Omega_{\varphi}$. These quantities are stored in a look-up table as a function of $\widetilde{c}$ for a given value of the flame filter size $\Delta$.

Tabulated data for the filtered progress variable balance equation are plotted in Fig. 2 for two different values of $\Delta / \delta_{l}$, where $\delta_{l}=D / S_{l}$ estimates the laminar flame thickness. Fig. 2(a) shows that when the filter size is larger than the flame thickness, as observed in realistic combustion LES, the contribution of the unresolved transport term $\Omega_{c}$ becomes as important as the filtered chemical reaction rate $\overline{\dot{\omega}}_{c}$ and therefore cannot be neglected.

\subsection{Laminar flame simulations}

1D and 2D laminar premixed flame simulations are performed to validate the present model and to determine its performances.

\subsection{1. $1 D$ premixed filtered laminar flames}

Steady 1D filtered laminar flames are computed to verify the capability of our model to reproduce both the correct flame front propagation speed and the filtered flame structure. Computations are performed 


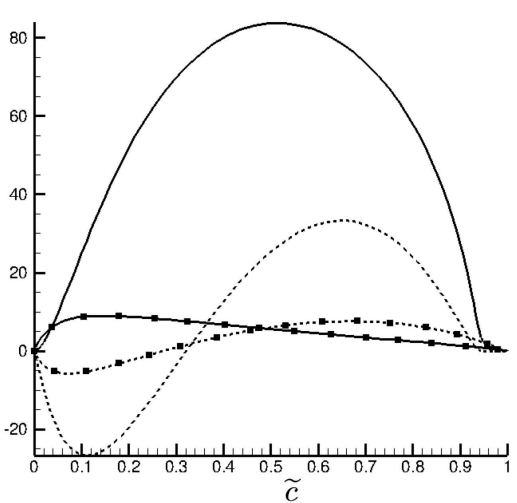

(a)

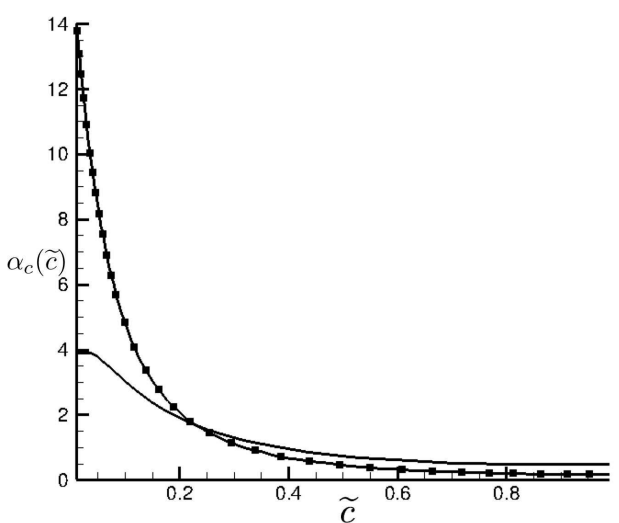

(b)

Figure 2. Tabulated data for the filtered progress variable balance equation. Without symbol: $\Delta / \delta_{l}=10$. Squares: $\Delta / \delta_{l}=100$. Left (a): $\overline{\dot{\omega}}_{c}$ (solid lines) and $\Omega_{c}$ (dashed lines) as a function of $\widetilde{c}$. Right (b): diffusion coefficient $\alpha_{c}$ as a function of $\widetilde{c}$

on uniform meshes with a grid spacing of $\Delta_{x}$. A parametric study is conducted for different filter sizes relative to the laminar flame thickness. For each case, a reference solution is obtained by filtering the $1 \mathrm{D}$ laminar premixed flame detailed chemistry solution. The simulations are initialized with the reference solution and the overall physical time for each run is $t_{\text {run }}=50 \Delta / S_{l}$.

A comparison between the numerical solutions on uniform mesh (solid lines) and the reference solution (dashed line) with $\Delta / \delta_{x}=50$ and for different values of $\Delta / \delta_{l}$ is first shown in Fig. 3(a). The predicted filtered progress variable profiles match the reference solution for all the filter size values. Note that as the thermo-chemical quantities are related in the FPI framework to the progress variable, the chemical flame structure is also recovered. Figure 4(a) shows that the predicted filtered front propagation speed $S_{\Delta}$ (squares symbols) remains very close to the true laminar flame speed for various values of $\Delta / \delta_{l}$. Triangular symbol in Fig. 4(a) represents simulation results with the approximation given by Eq. 10, i.e. $\alpha_{\varphi}=1$. This rough assumption leads to an under-prediction by a factor of three of the flame front propagation speed.

An important information for premixed combustion LES is the minimal number of grid points required to capture the filtered flame front without introducing numerical artifacts. The filtered flame front propagation speed is plotted as a function of the mesh resolution $\Delta_{x}$ in Fig.4(b). The flame speed is recovered with a good approximation for $\Delta / \Delta_{x} \geq 5$. Below this limit, numerical errors become important and the filtered flame front does not propagate at the correct speed. Then, for numerical reasons, the filter should be at least 5 times larger than the mesh size. Note that even approaches based on level-set transport that use sophisticated numerical methods to track the flame front position also require to filter the flame front at a scale larger than the mesh size in order to solve density gradients [12].

Finally a simulation has been performed without considering the filtering effect on the momentum equations, i.e. with $\alpha_{u}=1$ and $\Omega_{u}=0$ and is compared with the complete model solution in Fig. 3(b). For both simulations, density as well as velocity profiles match perfectly. In fact, the induced differences are transfered to the pressure that becomes a macro-pressure. As this macro-pressure remains very close to the static pressure, effects on the themodynamical state are very limited. Then in order to simplify the model implementation in 3D, the contribution corresponding to the filtering of a laminar flame in the momentum equation will be neglected. 


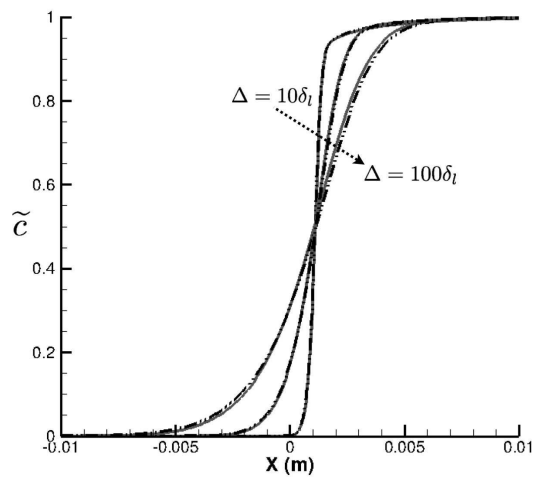

(a)

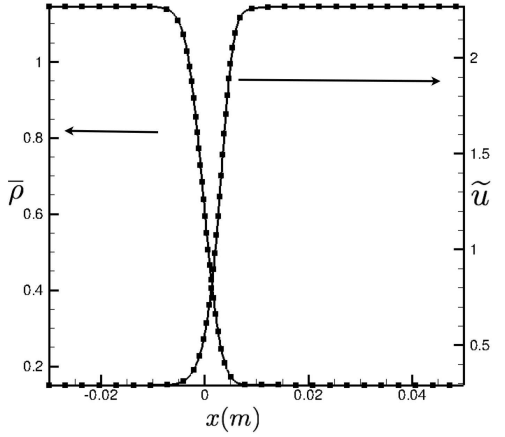

(b)

Figure 3. 1D filtered premixed flames solutions. Left (a): filtered progress variable (solid) compared to the reference solution (dashed) for $\Delta / \delta_{l}=10,50$ and 100. Right (b): effects of the flame filter in the momentum equation. Solid: $\alpha_{u}=1$ and $\Omega_{u}=0$. Symbols: $\alpha_{u}(\widetilde{c})$ and $\Omega_{u}(\widetilde{c})$ from the filtered database.

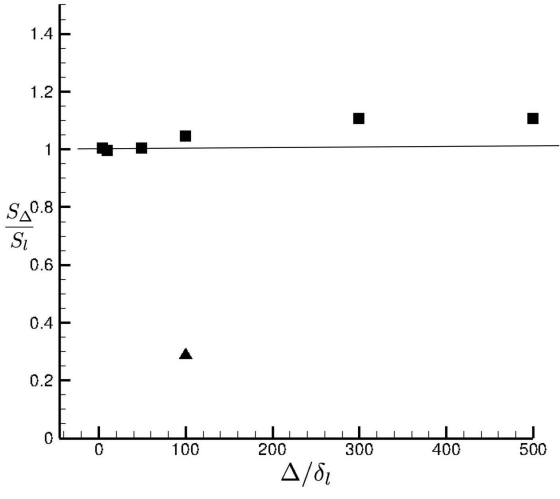

(a)

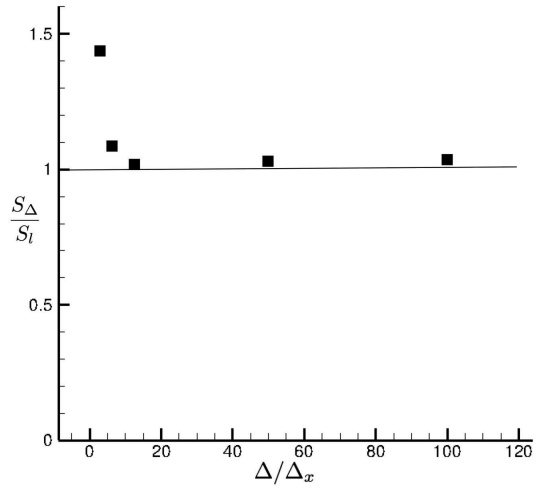

(b)

Figure 4. Predicted flame speed as a function of $\Delta / \delta_{l}$ (left) and $\Delta / \Delta_{x}$ (right). Squares symbols are the complete model solution and the triangle symbol is the solution with $\alpha_{\varphi}=1$.

\subsubsection{Flame vortex interactions}

A challenging test case for numerical combustion is the simulation of $2 \mathrm{D}$ flame-vortex interactions. This configuration that consists of a steady laminar premixed flame that interacts with a pair of counterrotating vortices convected by the flux of fresh gases, has been extensively numerically studied to evaluate combustion model performances [20,12]. A rectangular domain of size $10 \mathrm{~cm} \times 20 \mathrm{~cm}$ is meshed into a $256 \times 512$ grid. The initial locations of the clockwise and counterclockwise rotating vortices are $\left(x_{0}=\right.$ $\left.3.75 \mathrm{~cm} ; y_{0}=7.5 \mathrm{~cm}\right)$ and $\left(x_{0}=6.25 \mathrm{~cm} ; y_{0}=7.5 \mathrm{~cm}\right)$, respectively. The vortex intensity is $u^{\prime} / S_{l}=10.6$ and the vortex radius is $4 \mathrm{~cm}$. The mesh spacing $\Delta_{x}, 4$ times larger than the laminar flame thickness $\delta_{l}$, is representative of realistic combustion LES grid. The numerical simulation has been performed with the proposed model with a flame filter width $\Delta=100 \delta_{l}=25 \Delta_{x}$.

Iso-contours of progress variable are plotted at four instants in Fig. 5. These graphs show the deformation of the laminar front by the two vortices. The formation of the central cusp (Fig. 5(b) and Fig. 5(c)) 


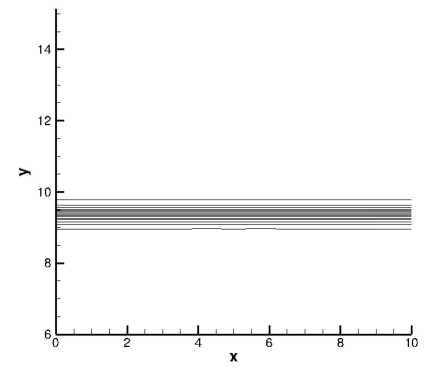

(a) $t=0 m s$

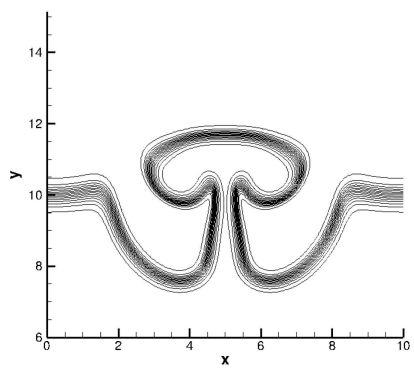

(c) $t=37.2 \mathrm{~ms}$

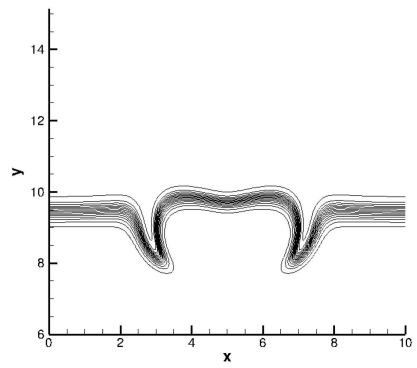

(b) $t=24.8 \mathrm{~ms}$

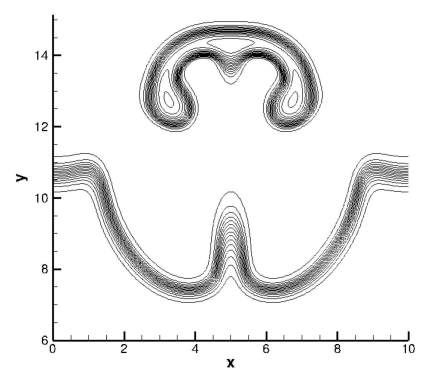

(d) $t=45.5 \mathrm{~ms}$

Figure 5. Iso-surfaces of progress variable for the flame vortex interaction configuration.

is due to the higher velocity in the zone between the vortices and the decrease of axial velocity on the exterior of the vortex pair. When the vortices pass through the flame, the high induced vorticity causes the formation of a pocket of fresh gas (Fig. 5(d)).

These results shows that the present model reproduces the flame front propagation for both planar and curved laminar flames when no flame wrinkling occurs at the subgrid scale. The extension to turbulent regimes is discussed in the following section.

\section{Turbulent filtered premixed flames modeling}

In practical turbulent combustion LES, the Gibson scale becomes generally larger than the filter size and flame front are wrinkled at the subgrid scale level. A strategy is proposed to extend the laminar model formulation to turbulent situations.

\subsection{Mathematical model}

Turbulent structures cause flame wrinkling that can increase the flame surface area at the subgrid scale. As a consequence the filtered flame front propagates at a turbulent flame speed $S_{t}$ [21] related to the laminar flame speed through the flame wrinkling factor $\Xi=S_{t} / S_{l}$.

The model is derived such that the filtered progress variable, that represents the filtered flame front, propagates at the turbulent flame speed $S_{t}$. The filtered flame thickness is assumed to be given by the 
filter size $\Delta$ and is not altered by small scale eddies.

Then, the filtered progress variable turbulent reaction rate is modeled by:

$$
\overline{\dot{\omega}}_{c_{t}}=\Xi \cdot \overline{\dot{\omega}}_{c}
$$

and the turbulent diffusion term is expressed as follow:

$$
\Omega_{c_{t}}=-(\nabla \cdot(\bar{\rho} \widetilde{\mathbf{u}} c-\bar{\rho} \widetilde{\mathbf{u}} \widetilde{c}))_{t}=\Xi \Omega_{c}+(\Xi-1) \nabla \cdot\left(\alpha_{c} \overline{\rho D} \nabla \widetilde{c}\right)
$$

The first term on the RHS corresponds to the thermal expansion and the second one models the turbulent fluxes. This formulation ensures that the filtered flame front propagates at the velocity $S_{t}$ in the normal direction because every RHS term of Eq. 20 has been multiplied by $\Xi$. The same procedure is applied to the energy equation.

\subsection{Summary of the model equations}

Finally to summarize, momentum, progress variable and energy equations can be written as follows:

$$
\begin{aligned}
\frac{\partial \bar{\rho} \widetilde{\mathbf{u}}}{\partial t}+\nabla \cdot(\bar{\rho} \widetilde{\mathbf{u}} \widetilde{u})= & -\nabla \cdot(\bar{P} \underline{\delta})+\nabla \cdot \tilde{\tau}+\nabla \cdot \bar{\tau}^{t} \\
\frac{\partial \bar{\rho} \widetilde{c}}{\partial t}+\nabla \cdot(\bar{\rho} \widetilde{\mathbf{u}} \widetilde{c})= & \Xi \nabla \cdot\left(\alpha_{c}(\widetilde{c}) \overline{\rho D} \nabla \widetilde{c}\right)+\Xi \Omega_{c}(\widetilde{c})+\Xi \overline{\dot{\omega}}_{c}(\widetilde{c}) \\
\frac{\partial \bar{\rho} \widetilde{E}}{\partial t}+\nabla \cdot(\bar{\rho} \widetilde{\mathbf{u}} \widetilde{E})= & -\nabla \cdot(\bar{P} \widetilde{u} \underline{\delta})+\Xi \Omega_{p}(\widetilde{c})+\nabla \cdot(\tilde{\tau} \tilde{\mathbf{u}})+\Xi \Omega_{E}(\widetilde{c}) \\
& +\Xi \nabla \cdot\left(\alpha_{E}(\widetilde{c}) \overline{\rho D} \nabla \widetilde{h}\right)+\Xi \bar{\omega}_{E}(\widetilde{c})
\end{aligned}
$$

Note that the effect of the flame filter $\Delta$ on the momentum equations is neglected and the subgrid scale turbulent fluxes $\nabla \cdot \bar{\tau}^{t}$ are modeled using the Wale model [22].

\subsection{LES of a turbulent premixed flame stabilized by a triangular flame holder}

This configuration, experimentally studied by Veynante \& Knikker [23] is a turbulent premixed flame stabilized by a triangular flame-holder. The height of the flame holder and the channel are $25 \mathrm{~mm}$ and $50 \mathrm{~mm}$, respectively. The burner is operated at atmospheric conditions with a stoichiometric propane/air mixture injected at $20 \mathrm{~ms}^{-1}$ that corresponds to a Reynolds number of 57000 based on the channel height.

In this paper preliminary results are presented. The computational domain made of 2.5 million hexahedral elements is identical to the mesh used by Moureau et al. [12]. A flat velocity profile is injected at the inlet of the domain and a constant atmospheric pressure is imposed at the outlet. For this preliminary simulation, the filter width is $\Delta=100 \delta_{l}$. Filtered tabulated chemistry data are stored in a one-dimensional look-up table. The subgrid flame wrinkling that appears in Eqs 25 and 26 can be either estimated from analytical models $[8,24,25,11])$ or from the solution of a surface density balance equation $[26,27]$. For the present computation, $\Xi$ has been estimated constant and equal to 3 .

A snapshot of the filtered flame front position plotted in Fig. 6 (top) shows the interactions between the resolved turbulence and the flame. The turbulence, generated in the shear layer right beyond the flame holder, causes flame wrinkling at the resolved scale, in particular at the downstream location. As all thermo-chemical data are related to $\widetilde{c}$, the post-processing of the filtered progress variable solution 

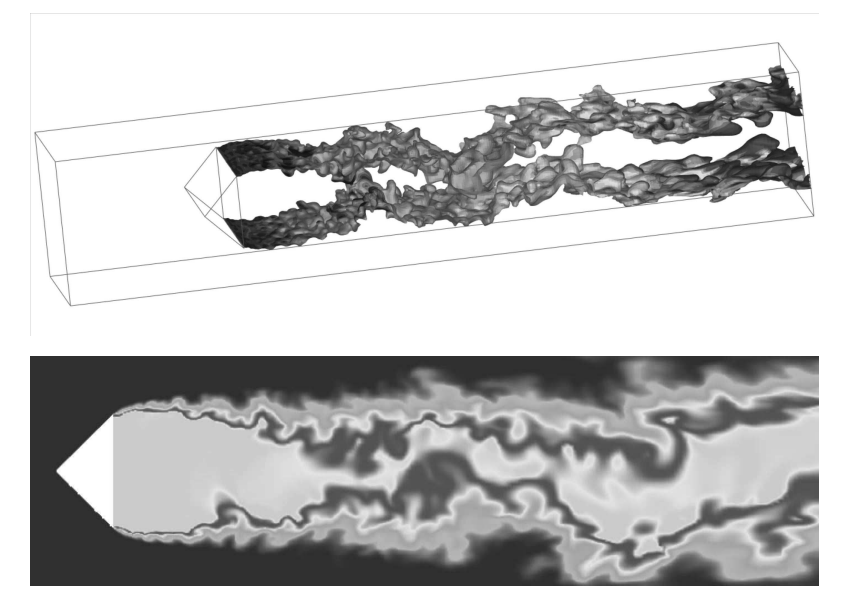

Figure 6. Iso surface of $\widetilde{c}=0.8$ (top) and contours of the $\mathrm{OH}$ mass fraction along the centerline plane (bottom)

with the filtered chemical database allows to access all chemical species. As an example, Fig.6 (bottom) shows contours of the $O H$ radical in the centerline plane.

This preliminary simulation shows that the model can be applied to realistic 3-D turbulent reactive flows. Further investigations and verifications, such as effects of filter width $\Delta$ and comparisons with mean experimental statistical data are required.

\section{Conclusion}

A new modeling strategy has been developed to introduce tabulated chemistry methods in premixed combustion LES. A 1D filtered laminar premixed flame is used to build a filtered chemical look-up table. The model shows good performances on 1D and 2D laminar flame computations. Finally the proposed strategy has been applied to perform a 3D computation of a turbulent premixed flame anchored by a triangular flame holder. Further investigations on this configuration, including comparison with experimental data and the introduction of a more accurate model for the subgrid scale flame wrinkling, are planned for the future.

\section{Acknowledgments}

The authors warmly acknowledge the support of the 2008 Summer Program of the Center for Turbulence Research (Stanford University - Nasa Ames) during which this work was initiated. We gratefully acknowledge Professors Vincent Moureau, Luc Vervisch and Heinz Pitsch for helpful discussions.

\section{References}

[1] U. Maas and S. Pope. Simplifying chemical kinetics: Intrinsic low-dimensional manifolds in composition space. Combust. Flame, 88:239-264, 1992.

[2] O. Gicquel, N. Darabiha, and D. Thévenin. Laminar premixed hydrogen / air counterflow flame simulations using flame prolongation of ildm with differential diffusion. In The Proceedings of the Twenty-Eighth Symposium (Int.) on combustion, pages 1901-1908. The Combustion Institute, Pittsburgh, 2000. 
[3] B. Fiorina, R. Baron, O. Gicquel, D. Thévenin, S. Carpentier, and N. Darabiha. Modelling non-adiabatic partiallypremixed flames using flame prolongation of ildm. Combustion Theory and Modelling, 7:449-470, 2003.

[4] J. A. van Oijen, F. A. Lammers, and L. P. H. de Goey. Modelling of complex premixed burner systems by using flamelet-generated manifolds. Combust. Flame, 127(3):2124-2134, 2001.

[5] L. Vervisch, R. Haugel, P. Domingo, and M. Rullaud. Three facets of turbulent combustion modelling: Dns of premixed flame, les of lifted nonpremixed v-flame and rans of jet-flame. J. of Turbulence, 5(4):1-36, 2004.

[6] B. Fiorina, O. Gicquel, L. Vervisch, S. Carpentier, and N. Darabiha. Premixed turbulent combustion modelling using tabulated chemistry and pdf. Proc. Combust. Inst., 30:867-874, 2005.

[7] T. D. Butler and P. J. O'Rourke. A numerical method for two-dimensional unsteady reacting flows. Proceedings of the 16th Symp. (Int.) on Combustion. The Combustion Institute, Pittsburgh, pages 1503-1515, 1977.

[8] O. Colin, F. Ducros, D. Veynante, and T. Poinsot. A thickened flame model for large eddy simulations of turbulent premixed combustion. Physics of Fluids, 12(7):1843-1863, 2000.

[9] M. Boger, D. Veynante, H. Boughanem, and A. Trouvé. Direct numerical simulation analysis of flame surface density concept for large eddy simulation of turbulent premixed combustion. In Twenty-Seventh Symposium (Int.) on Combustion, pages 917 - 925. The Combustion Institute, 1998.

[10] C Duwig. Study of a filtered flamelet formulation for large eddy simulation of premixed turbulent flames. Flow Turbulence and Combustion, 79(4):433-454, DEC 2007.

[11] H Pitsch. A consistent level set formulation for large-eddy simulation of premixed turbulent combustion. Combust. Flame, 143(4):587-598, DEC 2005.

[12] V. Moureau, B. Fiorina, and H. Pitsch. A level set formulation for premixed combustion LES considering the turbulent flame structure. COMBUSTION AND FLAME, 156(4):801-812, APR 2009.

[13] J. Galpin, A. Naudin, L. Vervisch, C. Angelberger, O. Colin, and P. Domingo. Large-eddy simulation of a fuel-lean premixed turbulent swirl-burner. Combust. Flame, 155:247-266, 2008.

[14] H Pitsch. Large-eddy simulation of turbulent combustion. Ann. Rev. Fluid Mech., 38:453-482, 2006.

[15] F GAO and EE OBRIEN. A LARGE-EDDY SIMULATION SCHEME FOR TURBULENT REACTING FLOWS. PHYSICS OF FLUIDS A-FLUID DYNAMICS, 5(6):1282-1284, JUN 1993.

[16] Avbp code : www.cerfacs.fr/cfd/avbp code.php and www.cerfacs.fr/cfd/ cfdpublications.html. 2008.

[17] O. Colin and M. Rudgyard. Development of high-order taylor-galerkin schemes for unsteady calculations. J. Comput. Phys., 162(2):338-371, 2000.

[18] T. Poinsot and S. K. Lele. Boundary conditions for direct simulations of compressible viscous flows. J. Comput. Phys., 1(101):104-129, 1992.

[19] R. J. Kee, J. F. Grcar, M. D. Smooke, and J. A. Miller. A fortran program for modelling steady laminar one-dimensional premixed flames. Technical report, Sandia National Laboratories, 1992.

[20] T. Poinsot, D. Veynante, and S. Candel. Quenching processes and premixed turbulent combustion diagrams. J. Fluid Mech., 228:561-605, 1991.

[21] T. Poinsot and D. Veynante. Theoretical and Numerical Combustion. R. T. Edwards, Inc., 2005.

[22] F Nicoud and F Ducros. Subgrid-scale stress modelling based on the square of the velocity gradient tensor. FLOW TURBULENCE AND COMBUSTION, 62(3):183-200, 1999.

[23] D. Veynante and R. Knikker. Comparison between les results and experimental data in reacting flows. J. of Turbulence, $7(35): 1-20,2006$.

[24] F. Charlette, C. Meneveau, and D. Veynante. A power-law flame wrinkling model for les of premixed turbulent combustion part i: non-dynamic formulation. Combust. Flame, 131(1/2):159-180, 2002.

[25] F. Charlette, C. Meneveau, and D. Veynante. A power-law flame wrinkling model for les of premixed turbulent combustion part i: dynamic formulation. Combust. Flame, 131(1/2):181-197, 2002.

[26] E. R. Hawkes and R. S. Cant. A flame surface density approach to large-eddy simulation of premixed turbulent combustion. Proc. Combust. Inst, 28:51-58, 2000.

[27] S. Richard, O. Colin, O. Vermorel, A. Benkenida, C. Angelberger, and D. Veynante. Towards large eddy simulation of combustion in spark ignition engines. Proc. Combust. Inst, 31:3059-3066, 2007. 